\title{
Intracranial Hemorrhage in Newborn with TAR Syndrome-A Feared Complication
}

\author{
Jório Brito Câmara ${ }^{\text {* }}$, Bruno Maciel Oliveira1, Larissa Ferreira Lima', Guilherme Straub Maia1, \\ Ana Paula Marques de Oliveira Melo1, Sergio Furlan1, Alessandra Di Marzio de Freitas Valle²
}

\author{
${ }^{1}$ Department of Radiology, Heliopolis Hospital, São Paulo, Brazil \\ ${ }^{2}$ Hospital Regional de Cotia, São Paulo, Brazil \\ Email: *joriocamara@gmail.com
}

How to cite this paper: Câmara, J.B., Oliveira, B.M., Lima, L.F., Maia, G.S., de Oliveira Melo, A.P.M., Furlan, S. and de Freitas Valle, A.D.M. (2020) Intracranial Hemorrhage in Newborn with TAR Syndrome-A Feared Complication. Open Journal of Medical Imaging, 10, 105-109. https://doi.org/10.4236/ojmi.2020.102010

Received: April 28, 2020

Accepted: June 8, 2020

Published: June 11, 2020

Copyright $\odot 2020$ by author(s) and Scientific Research Publishing Inc. This work is licensed under the Creative Commons Attribution International License (CC BY 4.0).

http://creativecommons.org/licenses/by/4.0/

\begin{abstract}
The thrombocytopenia-absent radius (TAR) syndrome is an autosomal recessive disease characterized by bilateral absence of radius with the presence of both thumbs and thrombocytopenia, many times associated with cardiac anomalies, intolerance or allergy to cow's milk and phocomelia. Imaging study is important for the correct diagnosis at birth and documentation of one of the diagnosis criteria (absence of radius) in prenatal care. The main hematologic symptoms and the most feared of them, intracranial bleeding, occurs when platelets levels are below $10,000 / \mathrm{mm}^{3}$, which is more common during the first months. Therefore, imaging study is crucial to quickly identify complications and correctly manage the case. In this case report, the patient had upper limps alterations at birth and at first week presented seizures, with transfontanellar ultrasound and head computed tomography without contrast demonstrating intracranial hemorrhage. Laboratory results and imaging review were able to diagnose TAR syndrome. The patient was treated with platelets transfusion and thrombocytopenia was solved. She is currently under specialized medical care, with no neurological deficits and showing satisfactory development.
\end{abstract}

\section{Keywords}

TAR Syndrome, Intracranial Hemorrhage, Neonatology, Transfontanellar Ultrasound, CT

\section{Introduction}

The thrombocytopenia-absent radius syndrome (TAR) is characterized by the absence of the radius and necessarily with the presence of the thumbs on both hands. It was described for the first time in 1959, having your diagnostic criteria 
established by Hall et al. 10 years after the first case report [1], despite the reports of newborns with thrombocytopenia and the absence of bones of the forearm exists since 1929 [2]. The incidence of the disease is still not yet established. However, the general projection of this index is around $0.5-1: 100,000$ live births [3].

The pattern of heredity, so as the physiopathology, is still not defined yet, been likely a hereditary autosomal inheritance, where the genes affected would be pathogenic variants of RMB8A. Case reports and genetic studies more currents suggest a strong cytogenetic association, especially the 1q21.1 deletion or microdeletion [4] [5].

The presence of thumbs bilaterally is important because it helps to separate from the main differential diagnoses like Fanconi anemia, Holt-Oram syndrome, VACTERL (vertebral anomalies, anorectal anomalies, cardiac anomalies, trachea-esophageal fistula, renal anomalies, limb anomalies) syndrome and trisomy 18 [6], since TAR syndrome can also present cardiac anomalies, such as atrial septal defect or limbs aplasia, phocomelia, gastrointestinal or renal anomalies.

Cow milk intolerance may be the most common anomaly that is not a diagnostic criterion, some authors suggesting an incidence around $47 \%$ to $62 \%$ [1] [3] among patients with TAR syndrome. The importance of this affection lays on the fact that the introduction or the maintenance of cow's milk may unleash hematologic symptoms, through mechanisms not well known and gastrointestinal symptoms as gastrointestinal bleeding.

Thrombocytopenia in this disease is megakaryocytic [1] [2] [7] and asymptomatic in most cases. However, in newborns, symptomatic cases can reach $90 \%$. Platelets serum level, especially at birth, is $15,000 / \mathrm{mm}^{3}$ to $35,000 / \mathrm{mm}^{3}$ [7]. When these values are below $10,000 / \mathrm{mm}^{3}$ [7] and cow's milk is introduced in the diet, the risk of bleeding is increased. Although most of hemorrhagic events are self-limited, intracranial bleeding is a serious complication and an important cause of impaired development.

Information was collected through medical record, imaging study obtained during hospitalization in Heliopolis Hospital and patient and family interview.

The lack of reports of bleeding events and the correct diagnosis makes the illustration of clinical data and imaging review relevant.

\section{Case Report}

Five days old patient, female, natural of the countryside of São Paulo, from a very low income family, is brought to the emergency room with history of three generalized tonic-clonic seizures during the last 24 hours. In physical examination, there were hypoplastic lower extremities (Figure 1(a) and Figure 1(b)), facial hemangioma and hypoplastic jaw. It was a full-term first-time twin pregnancy, without history of prenatal care or obstetric ultrasound. She was delivered via vaginal way, as a first newborn, whose sister died at birth. There was no history of consanguinity of the genitors. The parents did not have a known history of comorbidities or genetic disorders in the family. 
Laboratory tests showed leukocytosis at the expense of neutrophils, with no blasts and platelets level at $5,000 / \mathrm{mm}^{3}$. Kidney and liver functions were normal. HIV, VDRL and CMV tests were clear.

At first, the patient was managed with intravenous anti-convulsant drugs, with initial control of the seizures.

After the initial management, investigation was proceeded with upper limbs $\mathrm{x}$-ray that demonstrated bilateral radius absence with presence of both thumbs; and transfontanellar ultrasound revealed hyperechogenic areas in encephalic parenchyma (Figure 2(a) and Figure 2(b)), the largest measuring up to $2.3 \mathrm{~cm}$, compatible with intracranial hemorrhage.

Due to the recurrence of the seizures, head computed tomography was performed without contrast, in the attempt of discarding other structural causes, and showed hyperdense areas in the occipital region (Figure 3(a) and Figure 3(b)), confirming the bleeding alone.

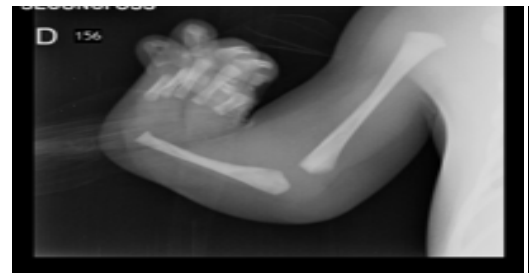

(a)

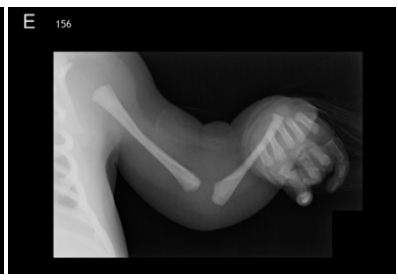

(b)

Figure 1. Anteroposterior $\mathrm{x}$-ray of the upper limps shows the absence of radius bilaterally with complete fingers, including thumbs, on both hands.

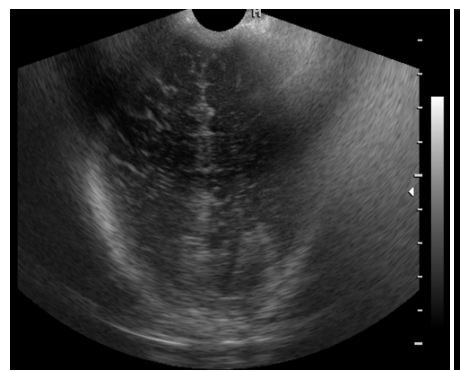

(a)

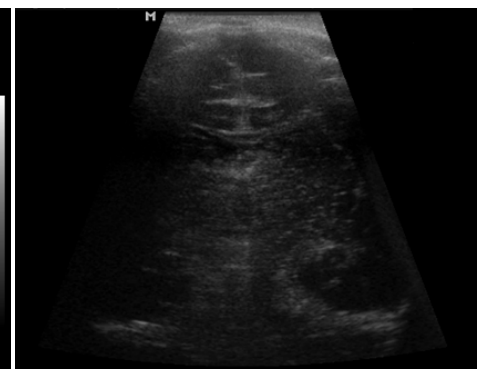

(b)

Figure 2. Coronal view of transfontanellar ultrasound demonstrated hyperechogenic foci in the interior of cerebral parenchyma, highly suggestive of bleeding.

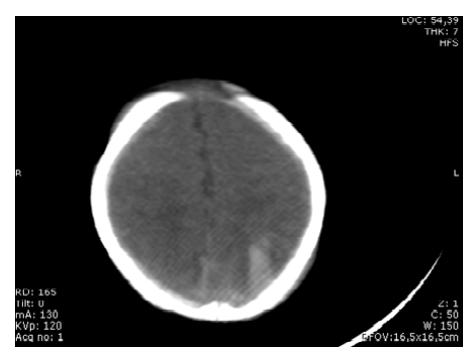

(a)

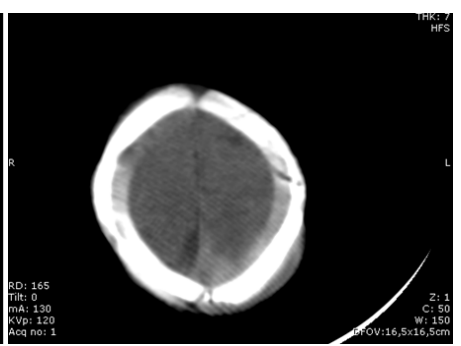

(b)

Figure 3. Head computed tomography revealed hyperdense sites on the left cerebral parenchyma and in the subdural space, confirming intracranial hemorrhage. 
Connecting physical exam, laboratory results and imaging study, and after discarding criteria for other diseases such as Fanconi Anemia and VACTERL alteration, the diagnostic criteria for TAR syndrome was fulfilled. Due to the formal indication (active bleeding with platelet level below $10,000 / \mathrm{mm}^{3}$ ), in absent radius with thrombocytopenia, treatment was initiated with platelets transfusion, demonstrating satisfactory response, reversion of hematologic status and complete reversion of tonic-clonic convulsions.

First neonatal and then bi-monthly follow up was performed during the first year of life of the patient, and although with poor prognostic due to the extensive intracerebral bleeding, the infant had a good development and did not have a new hemorrhagic event. Genetic testing was not performed in the follow up evaluations.

The patient is now under specialized medical care, with regular consultations with pediatrician, hematologist, orthopedic surgeon, neurology pediatrician and has no complications and appropriate neurological development.

\section{Discussion}

Anatomical changes observed at admission, confirmed with imaging study, and platelets levels were crucial to fulfill the diagnostic criteria of TAR syndrome. As reported in previous cases, secondary manifestations of thrombocytopenia, like petechial rash, dysentery [4] or central nervous system bleeding, are responsible for the diagnosis in several situations.

Despite being a rare syndrome, with incidence not well established, it is important the publication of reports of this pathology, since mortality rate in the first 12 months is the highest and prognosis is linked to the severity of thrombocytopenia in this period [8].

Diagnosis is possible at birth when skeletal changes and low level of platelets are noticed, allowing periodic following and decreasing of hemorrhagic events.

Scientific reports of this syndrome are spare in general publication, ALBINA, S. et al. [8] reported a case of TAR syndrome without intracranial bleeding at birth, evolving with increase of platelets levels at 18 months, making orthopedics procedures possible for better quality of life. Also, Cocci, P. et al. [6] described the case of three children with TAR syndrome. All of them were reported alive and underwent orthopedic, hematologic and nutritional follow-up. One of the patients had hemorrhagic diarrhea, but none had intracranial bleeding.

Health professionals must be ready to avoid unfavorable closures as hemorrhage and important anemia. The difficulty to perform venipuncture due to anatomic changes in these patients may delay intravenous infusion of crystalloid, blood product or drugs and intensify the pain, because of the various attempts to collect blood sample [6].

\section{Conclusion}

Articles and case reports about this subject are extremely relevant due to lack of 
scientific material regarding TAR syndrome and the need for early diagnosis, decreasing the incidence of unfavorable repercussions. It is important to spread among the medical community the information about a rare, but possible, cause of thrombocytopenia.

\section{Academic Attachment}

Hospital Heliopolis.

\section{Ethical Approval and Consent}

This article does not have trials with humans or animals performed by any of the authors. The patient parents were aware of the publication of the case and gave fully consent.

\section{Conflicts of Interest}

The authors declare no conflicts of interest regarding the publication of this paper.

\section{References}

[1] Greenhalgh, K.L., Howell, R.T., Bottani, A., Ancliff, P.J., Brunner, H.G., Verschuuren-Bemelmans, C.C., et al. (2002) Thrombocytopenia-Absent Radius Syndrome: A Clinical Genetic Study. Journal of Medical Genetics, 39, 876-881. https://doi.org/10.1136/jmg.39.12.876

[2] Adeyokunnu, A.A. (1984) Radial Aplasia and Amegakaryocytic Thrombocytopenia (TAR Syndrome) among Nigerian Children. The American Journal of Diseases of Children, 138, 346-348. https://doi.org/10.1001/archpedi.1984.02140420012005

[3] Naseh, A., Hafizi, A., Malek, F., Mozdarani, H. and Yassaee, V. (2012) TAR Syndrome: A Rare Case Report with Cleft Lip/Palate. The Internet Journal of Pediatrics and Neonatology, 14, No. 1.

[4] Omran, A., et al. (2012) Thrombocytopenia and Absent Radii (TAR) Syndrome Associated with Bilateral Congenital Cataract: A Case Report. Journal of Medical Case Reports, 6, Article No. 168. https://doi.org/10.1186/1752-1947-6-168

[5] Toriello, H.V. (2009) Thrombocytopenia Absent Radius Syndrome. In: Adam, M.P., Ardinger, H.H., Pagon, R.A., et al., Eds., GeneReviews, University of Washington, Seattle, 1993-2019. https://www.ncbi.nlm.nih.gov/books/NBK23758/

[6] Coccia, P., Ruggiero, A., Mastrangelo, S., et al. (2012) Management of Children with Thrombocytopeniaabsent Radius Syndrome: An Institutional Experience. Journal of Paediatrics and Child Health, 48, 166-169. https://doi.org/10.1111/j.1440-1754.2011.02069.x

[7] Hall, J.G. (1987) Thrombocytopenia and Absent Radius (TAR) Syndrome. Journal of Medical Genetics, 24, 79-83. https://doi.org/10.1136/jmg.24.2.79

[8] Silva, A., Morais, L., Rocha, C., Costa, E., Valente, E., Soares, P. and Barbot, J. (2001) Trombocitopenia e ausência de rádio (sindrome TAR): Caso clínico. Acta Pediatrica Portuguesa, 32, 47-50. 